\title{
Biochemical reactions for the removal of heavy metals from aqueous solutions
}

\begin{abstract}
In a previous study, lead $(\mathrm{Pb})$ and cadmium $(\mathrm{Cd})$ have been removed successfully from aqueous solutions using naturally occurring saponin isolated from Olea europaea and Citrus aurantium leaves. It was shown that biochemical removal of heavy metals was affected greatly by setting time, the concentration of heavy metal, concentration of saponin extract, and $\mathrm{pH}$ value. The process can easily remove heavy metals biochemically via coordinated complex formations between a central heavy metal and saponin as a natural bio-ligand. Accordingly, the effect of chemical factors is well-known but, the effect of physical factors is not. Our present work is focuses on the effect of some physical operational variables on the performance of saponin extraction for the removal of heavy metals. Temperature, agitation, and the synergism between them were the main physical factors that influence the biochemical reaction under investigation. Also, density, moisture content, and organic/inorganic contents have been determined for Olea europaea and Citrus aurantium leaves.
\end{abstract}

Keywords: biochemical removal, lead, cadmium, saponin extract, olea europaea, citrus aurantium
Volume 2 Issue 4 - 2018

\author{
Maher Mohamed Abed El-Aziza,' Mohamed \\ Ammar Khalifab ${ }^{2}$ \\ 'Chemistry Department, University of Tripoli, Libya \\ ${ }^{2}$ Chemistry Department, Azzaytuna University, Libya
}

\begin{abstract}
Correspondence: Maher Mohamed Abed El-Aziza, Faculty of Education/Gaser Ben Ghashir, Chemistry Department, University of Tripoli, Tripoli, Libya, Tel 002189243697 83, Email hrshr152@gmail.com
\end{abstract}

Received: June 30, 2018 | Published: July 16, 2018

\section{Introduction}

Olea europaea and Citrus aurantium are wild plants distributed in the Mediterranean region and grown since ancient time. Leaves of Olea europaea and Citrus aurantium contain so many biologically active ingredients such as fats/lipids, saponin, flavonoids, terpenoids etc. Occasionally, saponin was considered as a biological naturally occurring plant glycoside, characterized by their strong foaming properties in an aqueous solution that can be used as a ligand for decontamination of aqueous solutions due to its ability to form chemical complexes with heavy metals. ${ }^{1,2}$ Also, saponin has been applied as a surfactant having the complex-forming ability for determination of iron spectrophotometrically. ${ }^{3}$ The surfactant properties of saponin have been utilized as an important agent for heavy metal removal from aqueous wastes and remediation of contaminated sites. According to modern literature, saponins have served as a natural chelating agent to eliminate problems due to several heavy metals such as $\mathrm{Cr}, \mathrm{Cd}$, $\mathrm{Cu}, \mathrm{Pb}, \mathrm{Zn}$, and As from soil and wastewater. ${ }^{4-7}$ It has been shown that heavy metals were retained from its original solution due to some form of complexation with a carboxyl group (-COOH) in saponin. ${ }^{8}$ From a chemical point of view, the biochemical reaction between heavy metals and saponin represents a modern approach to (1) the synthesis of new organometallic complexes; (2) decontamination of industrial and radioactive effluents; (3) a non-traditional method for isolation of saponin from the plant kingdom. Our previous studies prove the presence of an oleanolic acid or hederagenin as an aglycone of triterpenoid saponin in Olea europaea and Citrus aurantium leaves as shown as in Figure 1. This means that there are at least two active centers (-OH and $-\mathrm{COOH}$ groups) coordinated directly with heavy metal atoms forming a complex. The concentration of the heavy metal, the concentration of saponin extract and $\mathrm{pH}$ were found the chemical operational variables affecting the biochemical reaction between saponin and heavy metal containing aqueous solutions. The present work aimed firstly to evaluate the biochemical reactions between saponin extracted from Olea europaea and Citrus aurantium leaves and lead/cadmium aqueous solutions under different physical environments. The effects of temperature, agitation, and synergism between them are the main operating factors under investigation affecting the reaction. Secondly, physicochemical characteristics of Olea europaea and Citrus aurantium leaves were determined to ensure the presence of a structure-behavior relationship.

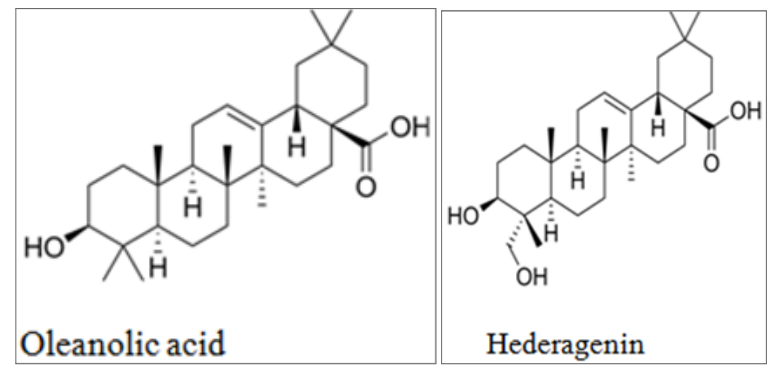

Figure I Chemical structure of Oleanolic acid and Hederagenin.

\section{Experimental}

\section{Materials and chemicals}

The leaves of Olea europaea (Oleaceae), and Citrus aurantium (Rutaceae) were collected during May 2017 from Tripoli region, Libya. The leaves of the two plants were cleaned with tap water followed by deionized water to remove dust and dried in shade at about $25 \pm 2^{\circ} \mathrm{C}$ and then cut into small pieces before extraction. Deionized water was used in all experimental investigations. Cadmium (II) nitrate tetrahydrate $\mathrm{Cd}\left(\mathrm{NO}_{3}\right)_{2} \cdot 4 \mathrm{H}_{2} \mathrm{O}$ was obtained from LANCASTER, ENGLAND. Lead (II) acetate $\left(\mathrm{CH}_{3} \mathrm{COO}\right)_{2} \mathrm{~Pb}$ is from SURE CHEM PRODUCTS LTD, ENGLAND. Petroleum ether (6080), n-hexane, methanol and absolute ethyl alcohol are from MERCK.

\section{Characterization of leaves}

The density of dry clean Olea europaea and Citrus aurantium leaves was determined by measuring the volume of a definite weight 
of three samples by water displacement method with the density calculated using the following equation:

$$
\operatorname{Density}(\rho)=m(g) / \Delta v\left(\mathrm{~cm}^{3}\right)
$$

The moisture content was determined by water loss method using a drying oven at $105^{\circ} \mathrm{C}$ for three samples of leaves and, $\%$ water content was calculated using equation [2] where, $\Delta W$ is the weight difference before and after complete dryness and, $W_{i}$ are the initial weight of leaves.

$$
\% \text { Moisure Cnotent }(M C)=\frac{\ddot{A} W}{W_{i}} \times 100 \text {... }
$$

The bio-organic content of non-moisturized leaves was determined by direct open combustion method using the non-luminous flame of benzene-burner for a sufficient time. After complete combustion, the weights of residues were measured and \% bioorganic content was calculated using the following equation:

$\%$ Bioorganic Content $(B O C)=\frac{\ddot{\mathrm{A}} W_{B O}}{W_{i}} \times 100$.

Where, $\Delta W$ is the weight difference before and after complete combustion of leave's material and, $W_{i}$ are the initial weight of leaves.
During combustion, bioorganic compounds are completely converted to $\mathrm{CO}_{2}$ and water vapor. After removing of moisture by evaporation and bioorganic compounds by combustion, the remaining residue is completely inorganic minerals which can be calculated from the following equations where, $W_{t}$ is the total weight of sample:

$$
\begin{gathered}
\text { Weight of Inorganic Minerals }\left(W_{\text {inorg. }}\right)=W_{t}-\left[W_{\text {mois. }}+W_{B O}\right] \ldots \ldots \ldots \ldots . . . . . . \\
\% \text { Inorganic Content }(I O C)=\frac{W_{\text {inorg. }}}{W_{t}} \times 100 \ldots \ldots \ldots \ldots \ldots . . . \ldots[5]
\end{gathered}
$$

\section{Physical parameters}

Preparation of crude extracts and heavy metal saponin complexes were carried out according to reference. ${ }^{1}$ Figure 2 summarizes the preparation steps of saponin extract solution from Olea europaea and Citrus aurantium leaves. The effect of temperature on the biochemical reaction between saponin extract and heavy metal aqueous solutions was carried out at different reaction temperatures $\left(15,40,60,80^{\circ} \mathrm{C}\right)$, then the volume and weight of wet and dried saponin complex were measured respectively. The effect of agitation was examined at different agitation time $(1,3,5 \mathrm{~min})$ under optimum conditions. Finally, synergism between temperature and agitation was tested for the reaction between lead $(\mathrm{Pb})$ and the saponin extract of Olea europaea.

Figure 2 Flowchart for preparation of saponin extract.

\author{
$500 \mathrm{~g}$ of clean dry leaves \\ $+$ \\ petroleum ether $\left(60-80^{\circ} \mathrm{C}\right)$ followed by $\mathrm{n}$-hexane then dry in air (Immersion for $24 \mathrm{~h}$ ) \\ $\downarrow$ \\ Dry leaves (without fats or lipids) \\ $+$ \\ $\sim 600 \mathrm{ml}\left(\mathrm{C}_{2} \mathrm{H}_{5} \mathrm{OH}+\mathrm{H}_{2} \mathrm{O}, \mathrm{v} / \mathrm{v}=\mathrm{I}: \mathrm{I}\right)$ in $2 \mathrm{~L}$ Pyrex beaker \\ $+$ \\ digestion for $6 \mathrm{~h}$ at $\mathrm{T}=55^{\circ} \mathrm{C}$ using thermostatic water bath \\ $\downarrow$ \\ Hot deep green color alcoholic/aqueous solution \\ $\downarrow$ \\ The extract solution was cooled and stored overnight at ambient temperature \\ $\downarrow$ \\ Decantation \\ $\downarrow$ \\ Extract solution $\sim 400 \mathrm{ml}$ \\ $\downarrow$ \\ Evaporation of alcohol at $\mathrm{T}=75^{\circ} \mathrm{C}$ for $30 \mathrm{~min}$. \\ $\downarrow$ \\ Concentrated Extract aqueous solution $\sim 200 \mathrm{ml}$ \\ $\downarrow$ \\ +ve test of saponin \\ $\downarrow$ \\ Undergo the biochemical reaction with heavy metals forming a complex precipitate
}




\section{Results and discussions}

\section{Physicochemical characteristics of leaves}

In this part of the present study, density $(\rho)$, percentage water content or moisture content (\%MC), percentage bioorganic content $(\% \mathrm{BOC})$ and percentage inorganic content $(\% \mathrm{IOC})$ for Olea europaea and Citrus aurantium leaves have been determined experimentally and the obtained results are presented in Table 1. From this data we found that the density for both Olea europaea and Citrus aurantium leaves is nearly the same having the density of pure natural water at $25^{\circ} \mathrm{C}$. For all samples, the density was found to be $\sim 1 \mathrm{~g} / \mathrm{cm}^{3}$. The above result may reflect the behavior of leaves when contacted with water where leaves neither floated nor immersed in water. This property decreases the chance for the leaves to directly remove heavy metals. Besides, the amount or concentration of free saponin is not enough in leaves. So, the use of a saponin extract is more favorable than the application of leaves for the removal of heavy metals from aqueous solutions. For the two last reasons, direct usage of plant leaves was excluded and not recommended for decontamination of aqueous solutions from heavy metals.

The percentage moisture content $(\% \mathrm{MC})$ for Olea europaea (triplicate) and Citrus aurantium (1 sample) leaves were determined practically. The weight of the samples was determined over 40minutes to obtain a constant weight. The weight of investigating samples is 10 , 15, and $20 \mathrm{~g}$ for Olea europaea and the weight of the Citrus Aurantium sample is $10 \mathrm{~g}$. The behavior of decreasing the weight of samples during heating at $105^{\circ} \mathrm{C}$ for $40 \mathrm{~min}$. is shown in Figure 3. From which it can conclude that for all samples, the weight was decreased along the examined time period (40min). The rate of moisture losses during heating were determined to be $82.5,130,165 \mathrm{mg} / \mathrm{min}$ for $10,15,20 \mathrm{~g}$ of Olea europaea samples and $70 \mathrm{mg} / \mathrm{min}$. for $10 \mathrm{~g}$ of Citrus aurantium sample respectively. As the weight of sample increased from 10 to $20 \mathrm{~g}$, the rate of moisture loss was increased from 82.5 to $165 \mathrm{mg} /$ min. It was found that for samples of the same weight $(10 \mathrm{~g})$ of both plants, the rate of loss of moisture from Olea europaea is more than that occurring from Citrus aurantium, and may be affordable to the biological, morphological and biochemical identity of individual plants. According to the percentage (\%) moisture content values in Table 1, the leaves of Olea europaea can be described as more moisturized than the leaves of Citrus aurantium. The main values of $\%$ water content are 33.5 and $28.0 \%$ for Olea europaea and Citrus aurantium leaves respectively.

Similarly through moisture content, bioorganic and inorganic contents could be evaluated in terms of percentage or gram weight. Complete chemical analysis of both Olea europaea leaves and Citrus aurantium leaves was summarized in Table 1 . We noticed that for all investigated samples, the amount of water stored in leaves, the amount of organic compounds, and the amount of inorganic minerals are nearly the same for both Olea europaea leaves and Citrus aurantium leaves. The minor differences between the two plants may reflect some kind of experimental, manipulation, or detection errors or may be attributed to the differences in biochemical nature of plants.

Olea europaea can be described as moisture and organic rich plant while, inorganic poor one compared to Citrus aurantium. The similarity in biochemical compositions of the two plants was confirmed by the obtained density data. The more similarity in biochemical composition, the nearest density will be and vice versa. Recall that saponin will be present as a bioorganic molecule in plant leaves, and hence the more bioorganic rich plant the high saponin content will be. ${ }^{9}$ Saponin containing organic compounds are easily oxidized by oxygen during direct combustion process, and it has been completely converted into carbon dioxide and water vapor. After complete mineralization of bioorganic compounds, dry inorganic residue/ash will be present in the porcelain crucible. In general, inorganic minerals are not greatly affected by elevated temperature due to its high thermal stability or resistance..$^{10}$

Table I Chemical analysis of Olea Europaea and Citrus Aurantium leaves

\begin{tabular}{lllll}
\hline \multirow{2}{*}{ Item } & \multicolumn{2}{l}{ Olea europaea leaves } & \multicolumn{2}{l}{ Citrus aurantium leaves } \\
\cline { 2 - 5 } & Weight(g) & Percentage (\%) & Weight(g) & Percentage (\%) \\
\hline Moisture & 3.3 & 33.6 & 2.8 & 28 \\
Bio-organics & 6 & 52.8 & 5 & 50 \\
Inorganics & 0.7 & 13.6 & 2.2 & 22 \\
Summation & 10 & 100 & 10 & 100 \\
Density & $1.00 \mathrm{~g} \cdot \mathrm{cm}^{-3}$ & & $1.05 \mathrm{~g}^{-\mathrm{cm}^{-3}}$ & \\
Test of saponin & $++\mathrm{ve}$ & & $+++\mathrm{ve}$ & \\
\hline
\end{tabular}

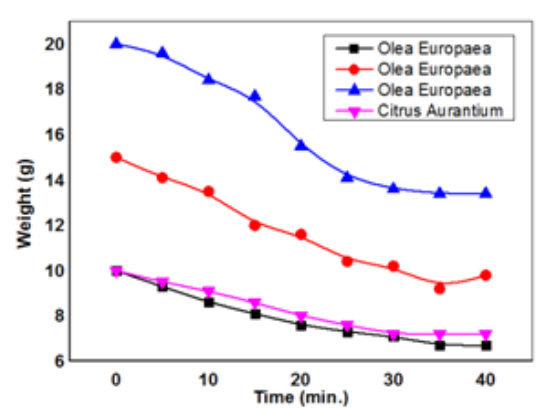

Figure 3 Decreasing behavior of weight of samples during heating at $105^{\circ} \mathrm{C}$ for $40 \mathrm{~min}$.

\section{Previous study}

Recently, previous work ${ }^{1}$ was published by our research group. The study detailed the effect of some chemical operational variables on performance of saponin with a variety of heavy metals. It has been shown that the chemical reaction between saponin and heavy metals was affected greatly by the following influences:

i. The type of heavy metal

ii. The concentration of heavy metals

iii. The concentration of saponin extract

iv. $\mathrm{pH}$ value of the medium

v. Setting time 
Aqueous and/or ethanolic extracts of Olea europaea and Citrus aurantium have been investigated as a test of saponin. According to Frothing test, Olea europaea and Citrus aurantium gave a positive response to the stable foam test, forming an emulsion with an ethanolic solution.

Ultraviolet/visible (UV/Vis.) analysis of different heavy metal saponin complexes exhibit major absorption peaks in the narrow wavelength range of $\sim 320$ to $400 \mathrm{~nm}$ with absorbance values in the range of 0.2 to 1.2 . Most of saponin itself has a normal absorption peak in the range of $\sim 250$ to $350 \mathrm{~nm}$, and hence the peaks of the prepared complexes were shifted towards longer wavelength (red shift), and this result may be due to a complex formation between saponin and heavy metals. The above mentioned results prove that saponin can easily separate and complex with heavy metals through the direct combination method. Fortunately, from an environmental point of view, heavy metals can easily be eliminated from its aqueous effluents by a natural saponin and may be lead to a more developed treatment method for so many hazardous waste streams. From the chemical point of view, a new class of organo-metallic complexes has been prepared successfully by the direct combination/chelation between heavy metal and natural saponin.

The obtained results show that the medium of the crude extract solution is weakly acidic $(\mathrm{pH}=5.16$ and 6.35$)$ possibly due to the triterpenoid saponin containing a carboxyl group $(-\mathrm{COOH})$ at carbon number 28. This supports the presence of triterpenoid saponin such as Oleanolic acid and Hederagenin. Six heavy elements $(\mathrm{Pb}, \mathrm{Cd}$, $\mathrm{Mn}, \mathrm{Co}, \mathrm{Ni}$, and $\mathrm{Zn}$ ) participated in the biochemical reaction with saponin but $\mathrm{Hg}$ and $\mathrm{Ag}$ failed along a wide/broad range of $\mathrm{pH}$ values (5.16-10.5) for both plants. The final volume of wet complexes after adequate setting times varied from the minimum volume $\approx 7 \mathrm{~mL}$ (for $\mathrm{Mn} \& \mathrm{Co}$ ) to the maximum volume $\approx 23 \mathrm{~mL}$ (for $\mathrm{Ni}$ ). The final setting time varied from 80 to $135 \mathrm{~min}$.

\section{The effect of temperature, agitation and synergism}

Our present studies were focused on the effect of temperature and agitation on the biochemical reaction between lead/cadmium and saponin as a natural bio-ligand. Also a synergism between the two affecting variables was observed to ensure that the reaction will proceed via optimum conditions. The effect of temperature on the biochemical reaction has been studied and the obtained results were shown in Figure 4.

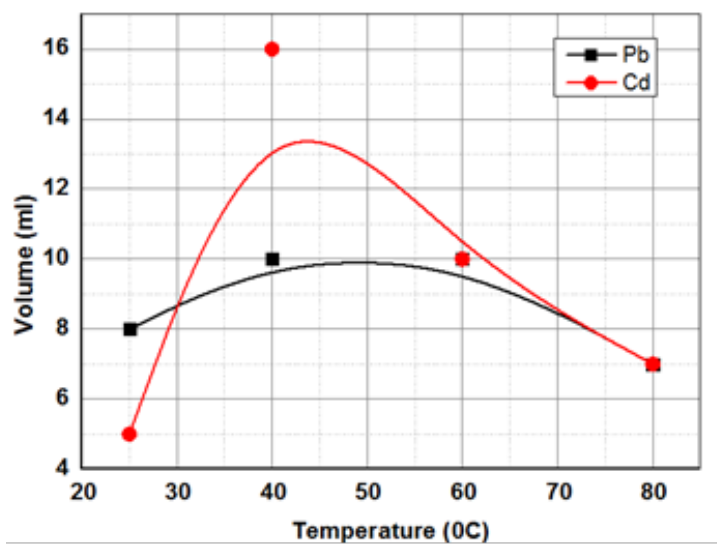

Figure 4 Temperature dependent of the reaction between $\mathrm{Pb} / \mathrm{Cd}$ and saponin.
As shown from this figure, the reaction was markedly improved within the temperature range of 40 to $50^{\circ} \mathrm{C}$ to reach a normal maximum at the middle center of the curve. This enhancement may be attributed to the increase in molecular effective collisions between the main reactants with elevated temperature at a microscopic level. ${ }^{11}$ Inhibition of the reaction starts near $50^{\circ} \mathrm{C}$ to reach the minimum steady state at $80^{\circ} \mathrm{C}$. According to the above mentioned results, it is highly recommended that the reaction should proceed at temperatures less than $50^{\circ} \mathrm{C}$. The reaction with cadmium seems to be more sensitive than lead towards temperature. In general the reaction is slightly affected by the temperature for lead.

The effect of agitation for $5 \mathrm{~min}$. on the biochemical reaction between heavy metal and saponin was studied and presented in Figure 5. This figure summarizes the obtained scientific fact that agitation was considered as the powerful operating factor affecting the reaction, where the reaction was enhanced continually as the agitation time increased, particularly for lead. For example, with lead the volume of the precipitate was increased from $8 \mathrm{~mL}$ without agitation (zero time) to about $33 \mathrm{~mL}$ at $5 \mathrm{~min}$. agitation period. The rate of improvement of biochemical reaction was equal to about $\approx 5 \mathrm{ml}$. $\mathrm{min}^{-1}$. The effectiveness of the reaction with cadmium is less than that with lead. This may be due to the effect of side reactions of cadmium with bioorganic compounds other than saponin. The amount of the products of the reaction was observed to be: $P b$-saponin $>C d$-saponin complex at the same agitation time.

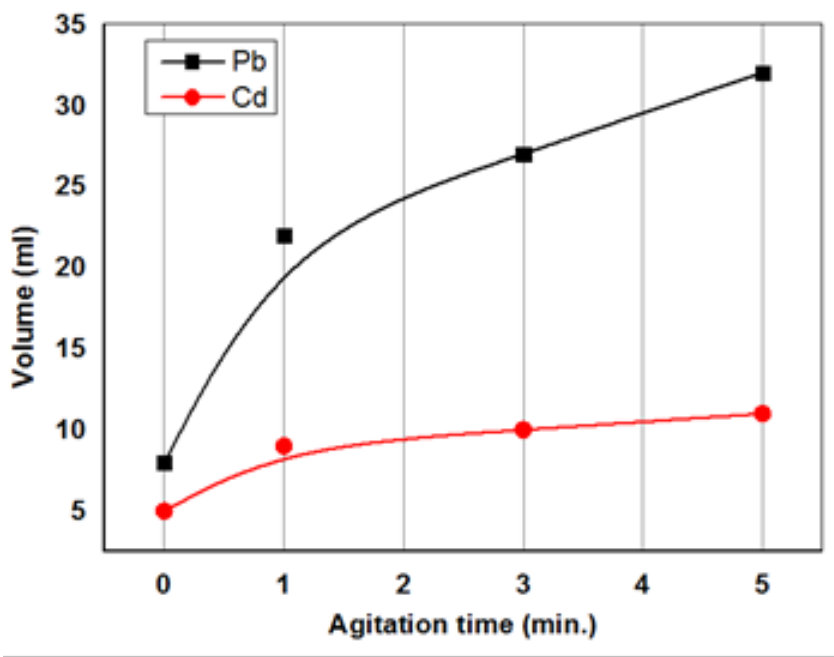

Figure 5 Agitation as a powerful operational variable.

A synergism effect may be defined as a simultaneously doubling of effects (at the same time) on the counter reaction. In a separate experiment, a simultaneously doubly effect of temperature and agitation was studied to know the optimum reaction conditions. The results of synergism effect on the reaction between lead and saponin to produce a complex were displayed as three dimensions (3D) in Figure 6 . From which we found that the reaction was slightly affected by synergism between temperature and agitation. This result may be explained by the thermal decomposition of the product during agitation process and the rate of formation of the product is not equal to the rate of decomposition leading to slightly synergistic effect. 


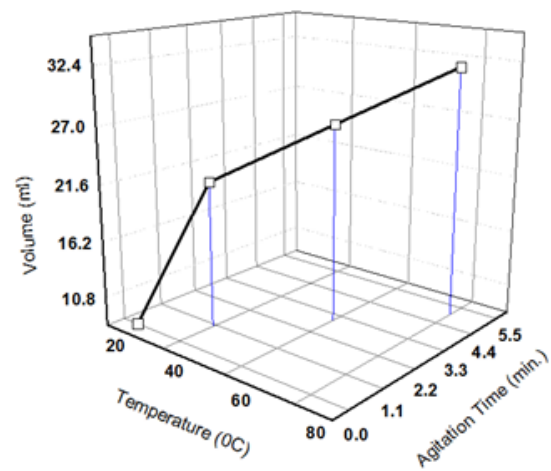

Figure $63 D$ presentation of synergism effect.

\section{Conclusion}

The removal of heavy metal from aqueous solutions by saponin extract has been established using biochemical reaction between them. The reaction under investigation was affected by means of physical factors such as temperature and agitation as well-as chemical factors such as concentrations, $\mathrm{pH}$, and setting time. It is highly recommended to apply water-saponin extract instead of leaves for the removal of lead and cadmium below $50^{\circ} \mathrm{C}$. A huge enhancement of the biochemical reaction can be obtained using agitation for $5 \mathrm{mins}$. Temperature, agitation can achieve the reaction separately but, the synergism between them leads to slight enhancements due to thermal decomposition of the product. The removal of cadmium $(\mathrm{Cd})$ using saponin is facilitated by temperature while, agitation facilitates the removal of lead $(\mathrm{Pb})$.

\section{Acknowledgments}

The authors would like to express appreciation and thanks to Maram Salem and Hadir Tareq, University of Tripoli, Libya for their valuable support in experimental work and laboratory setup.

\section{Conflict of interest}

The author declares that there are no conflicts of interest regarding the publication of this manuscript.

\section{References}

1. Abed El-Aziz MM, AshourAS, Madbouly HA, et al. Investigations on Green Preparation of Heavy Metal Saponin Complexes. $J$ Water Environ. Nanotechnol. 2017;2(2):103-111.
2. Romero-González J, Peralta-Videa JR, Rodríguez E, et al Potential of Agave lechuguilla biomass for $\mathrm{Cr}(\mathrm{III})$ removal from aqueous solutions: Thermodynamic studies. Bioresource Technology. 2006;97(1):178-182.

3. Yoshikazu Fujita, Itsuo Mori, Fujita K, et al. Approach to analytical chemistry of saponins: application of saponin as a surfactant having complex-forming ability to spectrophotometry of iron ion. Chem Pharm Bull. 1988;36(1):254-262.

4. Kilic E, Font J, Puig R, et al. Chromium recovery from tannery sludge with saponin and oxidative remediation. J Hazard Mater. 2011;185(1):456-462.

5. Hong KJ, Tokunaga S, Ishigami Y, et al. Extraction of heavy metals from MSW incinerator fly ash using saponins. Chemosphere. 2000;41(3):345-352.

6. Schmoll O, Howard G, Chilton J, et al. Protecting ground water for health: Managing the quality of drinking-water sources. World Health Organization. London UK: IWA Publishing; 2006.

7. Laura Silvia GV, José Bernardo PN, Fabian RM, et al. Surfactant Properties of the Saponins of Agave Durangensis, Application on Arsenic Removal. International Journal of Engineering and Applied Sciences. 2013;4(2):87-94.

8. Lidi Gao, Naoki K, Yuichi Sato, et al. Behavior and Distribution of Heavy Metals Including Rare Earth Elements, Thorium, and Uranium in Sludge from Industry Water Treatment Plant and Recovery Method of Metals by Biosurfactants Application. Bioinorganic Chemistry and Applications. 2012: Article ID 173819 .

9. Negi JS, Negi PS, Pant GJ, et al. Naturally occurring saponins: chemistry and biology. J Poison Med Plant Res. 2013;1(1):001-006.

10. Geoff Rayner-Canham, Tina Overton. Descriptive Inorganic Chemistry. $5^{\text {th }}$ ed. Freeman WH editor. New York: Clancy Marshall; 2010.

11. Temps F. Physical chemistry 3. Chemical kinetics. Lecture notes. 2014. 